\title{
Anterior Cervical Osteophytes Causing Dysphagia and Paradoxical Vocal Cord Motion Leading to Dyspnea and Dysphonia
}

\author{
Joon Won Seo, $\mathrm{MD}^{1}$, Ji Woong Park, $\mathrm{MD}^{1}$, Jae Chil Jang, $\mathrm{MD}^{2}$, Jae Wook Kim, $\mathrm{MD}^{3}$,
} Yang Gyun Lee, $\mathrm{MD}^{1}$, Yun Tae Kim, $\mathrm{MD}^{1}$, Seok Min Lee, $\mathrm{MD}^{1}$ Departments of ${ }^{1}$ Physical Medicine and Rehabilitation, ${ }^{2}$ Neurosurgery, and ${ }^{3}$ Otolaryngology-Head and Neck Surgery,
Soonchunhyang University College of Medicine, Seoul, Korea

Anterior cervical osteophytes are common and usually asymptomatic in elderly people. Due to mechanical compressions, inflammations, and tissues swelling of osteophytes, patients may be presented with multiple complications, such as dysphagia, dysphonia, dyspnea, and pulmonary aspiration. Paradoxical vocal cord motion is an uncommon disease characterized by vocal cord adductions during inspiration and/or expiration. This condition can create shortness of breath, wheezing, respiratory stridor or breathy dysphonia. We report a rare case demonstrating combined symptoms of dyspnea, dysphonia as well as dysphagia at the same time in a patient with asymptomatic anterior cervical osteophytes. Moreover, this is the first report demonstrating that anterior osteophytes can be a possible etiological factor for paradoxical vocal cord motion that induces serious respiratory symptoms.

Keywords Cervical anterior osteophyte, Paradoxical vocal cord motion, Dysphagia

\section{INTRODUCTION}

Anterior cervical osteophytes are frequently observed conditions in aged persons and are diagnosed on the basis of radiologic findings. Although they are asymptomatic in general, multiple symptoms may manifest due

Received July 26, 2012; Accepted October 11, 2012

Corresponding author: Ji Woong Park

Department of Physical Medicine and Rehabilitation, Soonchunhyang University Hospital, Soonchunhyang University College of Medicine, 59 Daesagwan-ro, Yongsan-gu, Seoul 140-743, Korea

Tel: +82-2-709-9370, Fax: +82-2-709-9265, E-mail: spotdoc88@gmail.com

() This is an open-access article distributed under the terms of the Creative Commons Attribution Non-Commercial License (http://creativecommons. org/licenses/by-nc/3.0) which permits unrestricted noncommercial use, distribution, and reproduction in any medium, provided the original work is properly cited.

Copyright $\odot 2013$ by Korean Academy of Rehabilitation Medicine to inflammation and edema arising from mechanical compressions. Although dysphagia due to anterior cervical osteophytes is not commonly reported [1], dysphagia accompanied by continual deterioration of dysphagia, dyspnea and dysphonia all together as seen in this case is very rare [2]. In fact, many physicians with different medical departments have failed to find the cause of this case.

We managed to diagnose our patient with anterior cervical osteophytes accidentally via a videofluoroscopic swallowing study (VFSS) while performing differential diagnosis through multiple tests as we mistakenly considered the osteophytes as brain lesions and progressive bulbar paralysis. In addition, we later confirmed the manifestation of paradoxical vocal cord motion through laryngendoscopy and observed that symptoms of dys- 
pnea and dysphonia manifested due to paradoxical vocal cord motion. Paradoxical vocal cord motion is a rare disease which refers to the vocal cords motion that illustrates asynchronization phenomenon in which the vocal cords is adducted rather than abducted in order to open the respiratory tract during inhalation or exhalation $[3,4]$. However, there have not been any cases of literary report on paradoxical vocal cord motion as a complication to anterior cervical osteophytes. In the current patient, we performed surgical removals of the osteophytes and confirmed immediate improvements of dysphagia and gradual improvements of dyspnea and dysphonia. Accordingly, we are reporting a case of anterior cervical osteophytes as the possible etiologic factor of paradoxical vocal cord motion along with literature review.

\section{CASE REPORT}

A 62-year-old male patient visited our hospital with dyspnea and mild dysphagia that began approximately 4 months prior to the visit as the main symptoms. Gentle exercise, such as steps, caused difficulty of inspiration 1 month ago, but it had become worse around the time of his visit, he appeared at rest and accompanied by dysphonia. Dysphagia emerged as foreign body sensation and intermittent aspiration during a solid meal, but he did not have history of weight loss or aspiration pneumonia. Various physicians, from a pulmonologist to an otorhinolaryngologist, failed to make an accurate diagnosis and the symptoms of the patient continued to progressively deteriorate.

The patient did not have any particular past internal or surgical medical history other than being a smoker for the last 20 years. The patient had clear consciousness without cognitive dysfunction at the time of his visit to our hospital. In physical examination, stridor was auscultated during inhalation. There was no abnormality of the tongue and no atrophy of the body muscles. The sensory tests displayed normal findings, and normal muscular strength was observed in all muscles of the upper and lower limbs on both sides in the manual muscular strength tests along with normal findings in the deep tendon reflex tests. Fibrillation was not observed in neurological examinations, and masticatory function and gag reflex were normal without observation of particular findings, such as dysarthria in linguistic assessments. Brain magnetic resonance imaging (MRI) for intracranial pathology did not show any abnormalities. There were no abnormal findings in the electrodiagnostic studies that were screened for neuromuscular diseases such as progressive bulbar paralysis. In addition, all the enzymes related to muscular diseases, such as creatine kinase, lactate dehydrogenase, and so on, were in the normal range of the laboratory test. Pulmonary function test was performed to find the cause of dyspnea, but normal pulmonary function was observed without abnormal findings, such as obstructive or restrictive pulmonary patterns. A VFSS showed that osteophytes were severely protruding at the anterior portion of the 5th to 7th cervical spine and translocated the lower pharynx and the upper esophagus anterosuperiorly. In the oral phase, there was no abnormality except for premature bolus loss. In the pharyngeal phase, although laryngeal elevation and epiglottic closures were normal and aspiration was not observed, a lowering of food took a circuitous route around the osteophytes and the pharyngeal transit time of the solid food was delayed by 1.4 seconds. X-ray and computerized tomography (CT) scan of the cervical vertebrae were performed to verify these anterior cervical osteophytes and revealed that the formation of large osteophytes was caused by overgrowth of cortical bones of the 5th to 7th cervical vertebrae. Osteophyte in the 5 th cervical spine was the most prominent and protruded $8 \mathrm{~mm}$ outside of the vertebral body. C-spine MRI showed anterior herniation of the intervertebral disc accompanied with a ruptured anterior longitudinal ligament, with fluid collection in the retropharyngeal space. A T2-weighted image showed high signal intensity with irregular enhancement caused by retropharyngeal irritation and inflammatory changes due to the large cervical osteophytes (Fig. 1). Anatomical deformities or mass-like lesions on the oropharynx and laryngopharynx were not observed in the larynx endoscopic examination. However, the paradoxical vocal cord motion, adducted vocal cords during the time of inhalation, was observed.

Because we did not find any possible etiology of these symptoms other than the anterior cervical osteophytes and the patient displayed a tendency of gradually aggravating dyspnea with severe anxiety and sleep disturbances, we decided to perform surgical treatments. Surgery was conducted through the anterior approach method. Removal of the anterior osteophytes and 5th to 6th cervical herniated disc were performed and interbody fixation with a bone graft was executed. 

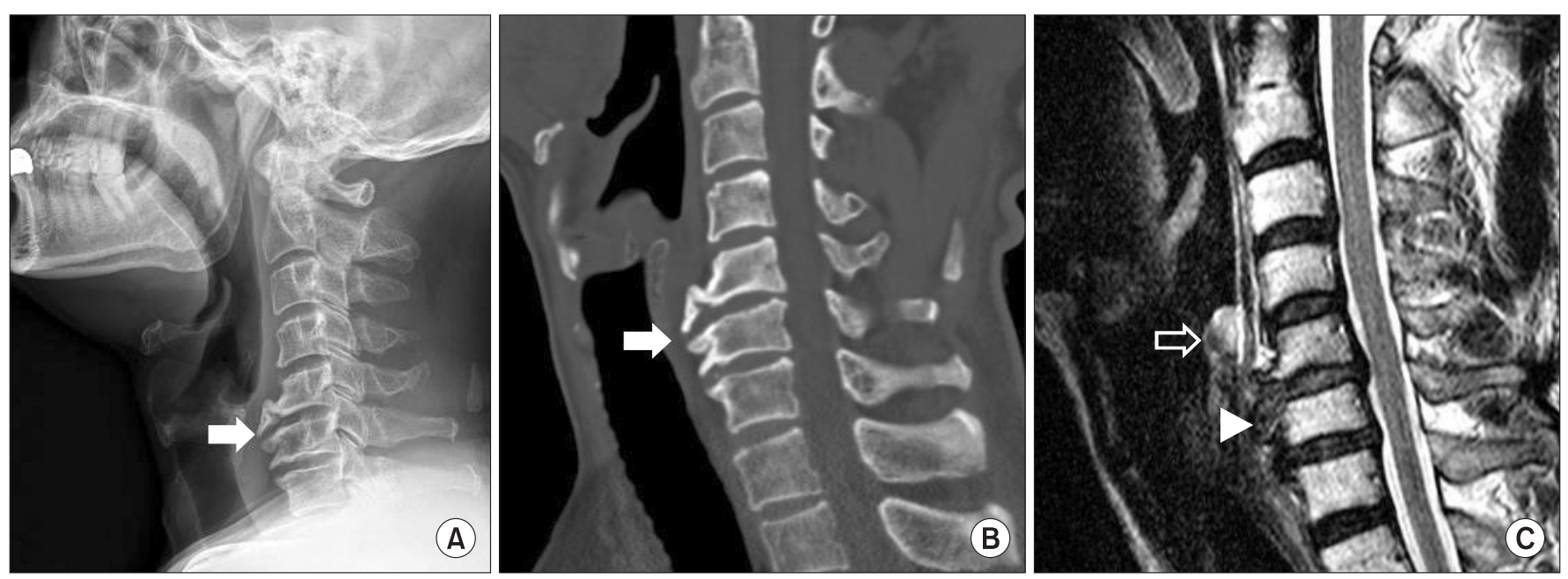

Fig. 1. Large anterior osteophytes (arrow) of the C5 and C6 vertebra bodies are demonstrated in the lateral views of the cervical spine X-ray (A) and the computed tomography scan (B). T2-weighted magnetic resonance image (C) shows an anterior extrusion of the C5-6 intervertebral disc (arrow head) and irregular high signal intensity (open arrow) in the retropharyngeal space.

Dysphagia disappeared immediately due to relief of mechanical pressure on the larynx, but dyspnea and paradoxical vocal cord motion still remained. Accordingly, for correcting the abnormal vocal cords motion, the patient performed the breathing exercises, such as relaxation technique and diaphragmatic breathing method. Finally, the respiratory difficulties and dysphonia were gradually improved after 3 months of continuous selftreatments.

\section{DISCUSSION}

Anterior cervical osteophyte is a common degenerative disease of the cervical vertebrae, and according to a study in Korea, this condition is frequently manifested in males with an average age of 66 years. In general, it is reported to occur in $2.9 \%$ to $4.1 \%$ of adults in Korea with its prevalence known to increase with age [5]. The most common vertebrae involved are C5 to C6 (40\%), followed by C4 to C5 (23\%), C3 to C4 (14\%), and C2 to C3 (14\%) [6].

Symptoms occur due to inflammation and edema induced by compression and friction of the surrounding organs arising from ossification of the anterior longitudinal ligament and the lateral ligament of the vertebrae. The most frequent symptom is dysphagia when consuming a solid diet and is experienced by approximately $28 \%$ of patients with anterior cervical osteophytes [7]. Other symptoms include dyspnea, hoarseness, dysphonia, and aspiration pneumonia, etc. and accompanied by infections of the respiratory system, emergency situations may arise which necessitate endotracheal intubations or incisions due to sudden respiratory failures. Such cases are considered to be the results of inflammation and edema in the mucous membrane of the pharynx [7]. In the present case, besides the direct bony compression, the inflammation and edema in the retro-laryngopharyngeal space was observed under MRI.

The diagnostic process was not easy in this case because the incidence is very rare which represents the simultaneous manifestation of dysphagia, dyspnea, and dysphonia in absence of musculoskeletal symptoms, such as neck pains. In the case of respiratory symptoms which are accompanied with dysphagia and dysphonia, neuromuscular causes, such as brain lesions, progressive bulbar paralysis, muscular dystrophy and amyotrophic lateral sclerosis can be distinguished. Thus, we performed multiple studies to detect nerve system abnormalities; however, we could not find any diseases in neurological examinations, neuroimaging studies, and electrodiagnostic studies.

In addition, if dyspnea is detected accompanied by stridor during respiration, chest X-ray, thoracic CT, pulmonary function test, and larynx endoscopic should be performed for the possibility of obstructing the upper respiratory tract. However, there were no specific findings for all the tests in the present case. This seemed to be the 
result when placing main focus on the mechanical causes rather than the functional assessments on the vocal cords movement.

There has not been any literary report on paradoxical vocal cord motion associated with multiple complications of anterior cervical osteophytes. Paradoxical vocal cord motion refers to asynchronized motion in which the vocal cord is adducted rather than abducted when the respiratory tract opens during inhalation or exhalation [3]. Due to this, dyspnea and dysphonia accompanied by stridor occur, and the condition is frequently misdiagnosed as intractable asthma in adults. Although the causes are not yet clear, asthma, refluxing esophagitis, viral infection of central nerve system, surgical complications, and neuropsychological factors are considered to be the relevant factors [4].

In the event of manifestation of dyspnea due to anterior cervical osteophytes, obstructive patterns of the pulmonary function [7] and prominences on the rear surface of the larynx under laryngendoscopy are usually observed [8]. However, we later found out that functional closure of the respiratory tract was due to paradoxical vocal cord motion.

Treatment of anterior cervical osteophytes can be divided into conservative and surgical methods [9]. Although inflammation and edema can be reduced with antibiotics, anti-inflammatory drugs, steroids, and muscle relaxants if the symptoms are mild, they are not fundamental treatments of osteophytes itself. In cases of severe reduction in body weight or no response to the medications, surgical treatment is required [10]. In our case, we chose surgery because the patient displayed a trend of gradual progression of dyspnea along with a growing sense of anxiety. Although dysphagia was immediately improved after the surgery along with resolved symptoms of direct compression of the esophagus, the paradoxical vocal cord motion which caused dyspnea and dysphonia persisted for quite some time even after the eliminations of structural compression. This is thought to be the result of acquired fear and abnormal functional adaptation to the changed vocal cords motion over a prolonged period of time. However, the symptoms were improved through the continuous stress alleviation method and the abdominal breathing technique.

If dysphagia, dyspnea and dysphonia existed simulta- neously, anterior cervical osteophytes must be checked through radiological evaluations of the cervical vertebrae as well as careful inspections of vocal cords movement through larynx endoscopic examinations.

\section{ACKNOWLEDGMENTS}

This work was supported by the Soonchunhyang University Research Fund.

\section{REFERENCES}

1. Granville LJ, Musson N, Altman R, Silverman M. Anterior cervical osteophytes as a cause of pharyngeal stage dysphagia. J Am Geriatr Soc 1998;46:1003-7.

2. Ahn YJ, Hahn SH, Yang BK, Yi SR, Yoo JH, Yoon DJ, et al. Diffuse idiopathic skeletal hyperostosis associated with dysphonia and dysphagia: a case report. J Korean Soc Spine Surg 2006;13:327-31.

3. Forrest LA, Husein T, Husein O. Paradoxical vocal cord motion: classification and treatment. Laryngoscope 2012;122:844-53.

4. Karaman E, Duman C, Alimoglu Y, Isildak H, Oz F. Paradoxical vocal cord motion: haloperidol usage in acute attack treatment. J Craniofac Surg 2009;20:16024.

5. Kim SK, Choi BR, Kim CG, Chung SH, Choe JY, Joo $\mathrm{KB}$, et al. The prevalence of diffuse idiopathic skeletal hyperostosis in Korea. J Rheumatol 2004;31:2032-5.

6. Gamache FW Jr, Voorhies RM. Hypertrophic cervical osteophytes causing dysphagia: a review. J Neurosurg 1980;53:338-44.

7. Demuynck K, Van Calenbergh F, Goffin J, Verschakelen J, Demedts M, Van de Woestijne K. Upper airway obstruction caused by a cervical osteophyte. Chest 1995;108:283-4.

8. Kapetanakis S, Vasileiadis I, Papanas N, Goulimari R, Maltezos E. Can a giant cervical osteophyte cause dysphagia and airway obstruction? A case report. Wien Klin Wochenschr 2011;123:291-3.

9. Faruqi S, Thirumaran M, Blaxill P. An osseous cause of dysphagia. Med J Aust 2008; 188:671.

10. Marra A, Dario A, Scamoni C, Pozzi M, Soldati M, Dorizzi A. Dysphagia due to anterior cervical osteophyte: case report. J Neurosurg Sci 1991;35:229-31. 